\title{
Electronic Structure and X-Ray Magnetic Circular Dichroism in $\mathrm{Sm}$-Doped $\mathrm{Bi}_{2} \mathrm{Se}_{3}$
}

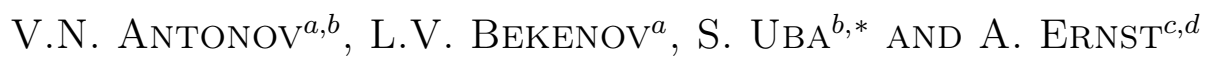 \\ ${ }^{a}$ G.V. Kurdyumov Institute for Metal Physics of the N.A.S. of Ukraine, 36 Vernadsky Street, 03142 Kiev, Ukraine \\ ${ }^{b}$ Faculty of Mathematics and Informatics, University of Bialystok, \\ K. Ciolkowskiego 1M, PL-15-245 Bialystok, Poland \\ ${ }^{c}$ Max-Planck Institut für Mikrostrukturphysik, Weinberg 2, D-06120 Halle \\ ${ }^{d}$ Institut für Theoretische Physik, Johannes Kepler Universität, A 4040 Linz, Austria
}

\begin{abstract}
We studied the structural, electronic, and magnetic properties of Sm-doped $\mathrm{Bi}_{2} \mathrm{Se}_{3}$ using a GGA approximation in a frame of the fully relativistic spin-polarized Dirac linear muffin-tin orbital band-structure method. The Xray absorption spectra (XAS) and X-ray magnetic circular dichroism at the $\operatorname{Sm} M_{4,5}$ edges were investigated theoretically from first principles. The calculated results are in good agreement with experimental data. The complex fine structure of the $\mathrm{Sm} M_{4,5}$ XAS in $\mathrm{Sm}$-doped $\mathrm{Bi}_{2} \mathrm{Se}_{3}$ was found to be not compatible with a pure $\mathrm{Sm}^{3+}$ valency state. The interpretation demands mixed valent states.
\end{abstract}

DOI: 10.12693/APhysPolA.133.453

PACS/topics: 75.50.Cc, 71.20.Lp, 71.15.Rf

\section{Introduction}

One of the most striking discoveries in recent years which has emerged from research into spin-orbit coupling is a new state of matter known as a topological insulator (TI) [1]. Topological insulators are fully gapped in the bulk but, due to strong spin-orbit coupling, possesses one or more robust metallic surface states which are protected by time-reversal symmetry (TRS). Typically, the Dirac point, the tip of the cone, is located a few hundred $\mathrm{meV}$ below the Fermi energy $E_{F}$ and the bulk conduction band crosses $E_{F}$. Beyond the inherent importance of exploring a complex phase of quantum matter, these systems are of great interest for device applications involving quantum computing and photonics [2].

It is important to break the TRS of TIs to realize novel physical phenomena such as, for example, carrier-independent long-range ferromagnetic order, giant magneto-optical Kerr effect or the newly discovered quantum anomalous Hall effect (QAHE) [3]. The QAHE is observed in magnetic TIs where quantized transport occurs in the absence of an external magnetic field and thus discrete Landau levels. It can be achieved by inducing ferromagnetism in the TI. Ferromagnetic ordering has been successfully achieved in $3 \mathrm{D}(\mathrm{Bi}, \mathrm{Sb})_{2}(\mathrm{Se}, \mathrm{Te})_{3}$ type TIs by doping with $3 d$ transition metals, such as Mn and $\mathrm{Cr}[4,5]$. In general, however, doping with transition metals has the intrinsic disadvantage that a divalent ion replaces the trivalent $\mathrm{Bi}$ due to hybridization. Doping with rare-earth (RE) elements, on the other hand, leads to an isoelectronic and substitutional doping on the $\mathrm{Bi}$

*corresponding author; e-mail: uba@uwb.edu.pl site. Besides, RE ions offer the advantages of larger magnetic moments. Recently Chen et al. [6] report the successful preparation of a series of $\left(\mathrm{Sm}_{x} \mathrm{Bi}_{1-x}\right)_{2} \mathrm{Se}_{3}$ crystals magnetic TIs, which exhibit ferromagnetism up to about $52 \mathrm{~K}$. All evidence suggests that $\mathrm{Sm}$-doped $\mathrm{Bi}_{2} \mathrm{Se}_{3}$ is a candidate for high-mobility magnetic TI. Here we present the theoretical calculations of the X-ray absorption spectra (XAS) and X-ray magnetic circular dichroism (XMCD) of Sm-doped $\mathrm{Bi}_{2} \mathrm{Se}_{3}$ from first principles. We found that the complex fine structure of the $\operatorname{Sm} M_{4,5}$ XAS in $\mathrm{Sm}$-doped $\mathrm{Bi}_{2} \mathrm{Se}_{3}$ is not compatible with a pure $\mathrm{Sm}^{3+}$ valence state. The interpretation demands mixed valent states.

\section{Computational details}

$\mathrm{Bi}_{2} \mathrm{Se}_{3}$ is a narrow band gap semiconductor with a tetradymite crystal structure with the space group $D_{3 d}^{5}$ $(R \overline{3} m)$, number 166 . This rhombohedral layered structure is formed by five atomic layers as a basic unit cell, named a quintuple layer (QL) (see Fig. 1a). The interlayer bonding within the QLs is strong because of the dominant covalent character, but the bonding between the QLs is much weaker due to the van der Waals-type interaction.

The details of the computational method are described in our previous papers [7-12]. Band structure calculations were performed using the fully relativistic linear muffin-tin orbital (LMTO) method [13] with exchangecorrelation functional of a GGA-type [14]. This implementation of the LMTO method uses four-component basis functions constructed by solving the Dirac equation inside an atomic sphere [15], which is crucial for a correct description of $p_{1 / 2}$ states of heavy elements such as $\mathrm{Pb}$ or $\mathrm{Bi}[16]$. 


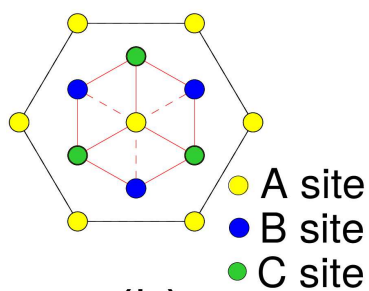

(b)

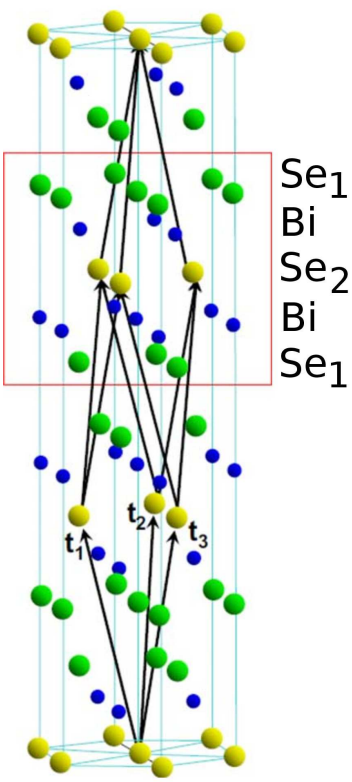

(a)

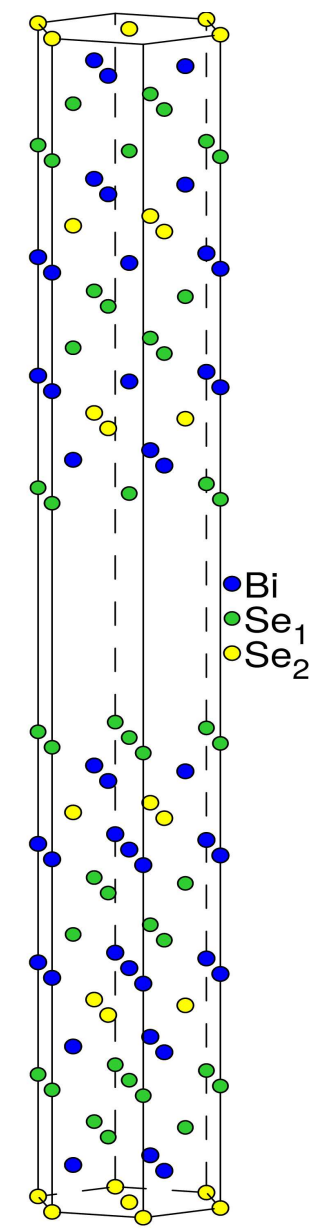

(c)

Fig. 1. Crystal structure of the $\mathrm{Bi}_{2} \mathrm{Se}_{3}$ compound. (a) The hexagonal supercell containing 15 atomic layers; $\boldsymbol{t}_{1,2,3}$ are primitive translation vectors. The quintuple layer is shown in the red box. (b) The top view of a QL in the triangle lattice. Three sets of different sites, labeled as A, B and C sublattices, respectively, are presented. (c) Crystal structure of the $\mathrm{Bi}_{2} \mathrm{Se}_{3}$ (111) thin film with the thickness of six quintuple layers with one quintuple replaced by empty spheres.

The electronic structure of surface states was investigated for crystal structure of the $\mathrm{Bi}_{2} \mathrm{Se}_{3}$ (111) thin film with the thickness of six QLs with one quintuple replaced by empty spheres (see Fig. 1(c)). The inter-layer bonding between two QLs is much weaker than that inside the $\mathrm{QL}$; it is natural to expect that the cleavage plane should be between two QLs.

To investigate the electronic and magnetic properties of bulk $\mathrm{Bi}_{2} \mathrm{Se}_{3}$ with dilute Sm doping, a $(2 \times 2 \times 1)$ supercell containing $24 \mathrm{Bi}$ and 36 Se atoms was employed. We use the experimental lattice constants $a=4.138 \AA$ and $c=28.640 \AA$ with internal parameters $\nu=0.206$ and $\mu=0.399[17]$. We consider a single Bi atom replaced by a Sm atom. Such a dopant concentration is 4.17 at\% $(x=0.0417)$. The $\mathrm{X}$-ray absorption and dichroism spectra were calculated taking into account the exchange splitting of core levels.

\section{Results and discussion}

Figure 2 presents the $a b$ initio calculated band structure of the $\mathrm{Bi}_{2} \mathrm{Se}_{3}$ (111) thin film with the thickness of five quintuple layers for non-relativistic approximation (b) and fully relativistic Dirac approximation (c). The surface states are indicated by red lines while the other lines correspond to the bulk bands. Nonrelativistic calculations produce rather large energy band gap at the Fermi level of around $0.54 \mathrm{eV}$. In fully relativistic Dirac approximation (Fig. 2c) two chiral surface states are clearly seen to connect the conduction band and valence band to form a single Dirac-type contact at the $\Gamma$ point and aligning with the Fermi energy. Our calculations are in good agreement with previous calculations [18, 19]. (a)

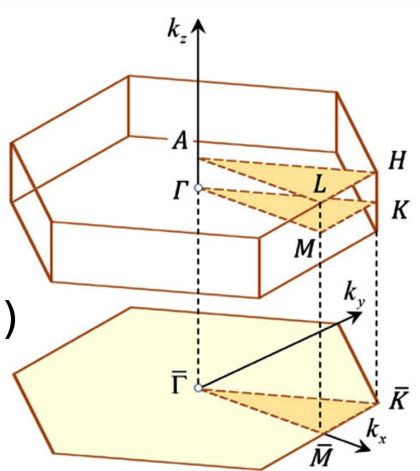

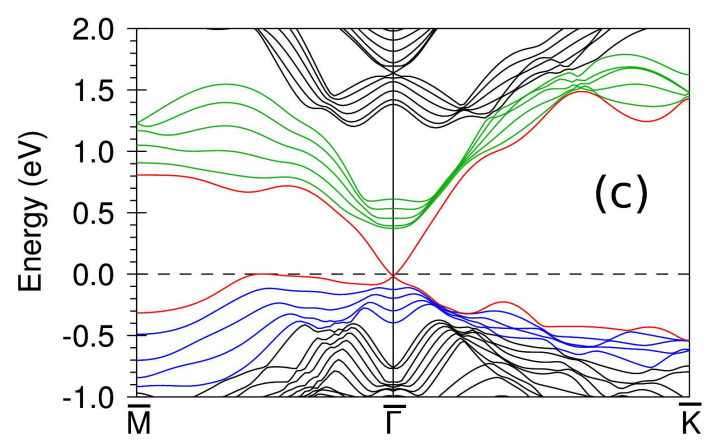

Fig. 2. (a) The 3D first Brillouin zone and the corresponding surface $2 \mathrm{D}$ BZ below with the high symmetry points indicated. The ab initio calculated band structure of the $\mathrm{Bi}_{2} \mathrm{Se}_{3}$ (111) thin film with the thickness of six quintuple layers for non-relativistic approximation (b) and fully relativistic Dirac approximation (c); the red lines indicate the surface states while the other lines correspond to the bulk bands. 


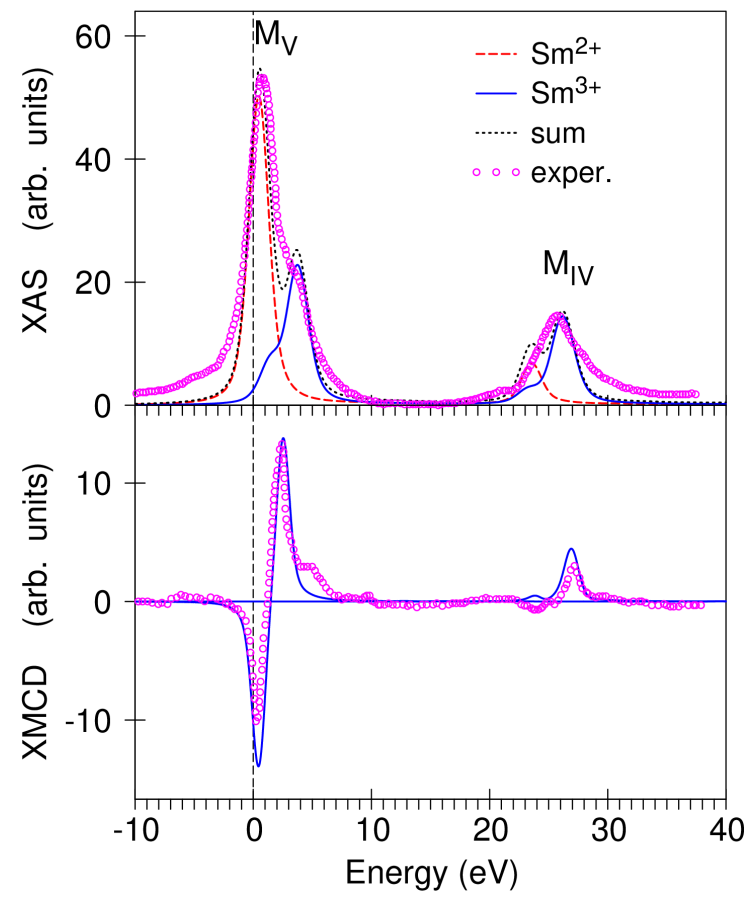

Fig. 3. Top panel: the X-ray $M_{4,5}$ absorption spectra (open circles) in $\left(\mathrm{Sm}_{0.05} \mathrm{Bi}_{0.95}\right)_{2} \mathrm{Se}_{3}$ [6] compared with the theoretically calculated ones (dotted black curves); the spectra for trivalent (full blue line) and divalent (dashed red curve) Sm ions are presented separately; lower panel: the XMCD experimental spectrum (open circles) in $\left(\mathrm{Sm}_{0.05} \mathrm{Bi}_{0.95}\right)_{2} \mathrm{Se}_{3}$ at the $\mathrm{Sm} M_{4,5}$ edges [6] and the theoretically calculated one for trivalent $\mathrm{Sm}^{3+}$ ions (full blue lines).

Figure 3 (upper panel) shows the calculated XAS spectra in the GGA approximation together with the experimental spectra measured by Chen et al. [6]. The complex fine structure of the $\mathrm{Sm} M_{4,5}$ XAS in Sm-doped $\mathrm{Bi}_{2} \mathrm{Se}_{3}$ was found to be not compatible with a pure $\mathrm{Sm}^{3+}$ valence state. The interpretation demands mixed valent states. The divalent $\mathrm{Sm}$ solution contributes to the major peak at the $\mathrm{Sm} M_{5}$ edge (dashed red curve), a high energy shoulder is mostly due to the trivalent solution (full blue line).

The dichroism at the $M_{5}$ edge has two lobes, a negative and a positive one. The positive lobe is larger in comparison with the negative. $\mathrm{Sm}^{2+}$ solution is nonmagnetic, therefore the XMCD spectra at the $M_{4,5}$ are completely due to $\mathrm{Sm}^{3+}$ ions. The theory relatively well describes the shape and intensity of the XMCD spectra at the $M_{4,5}$ edges, however, slightly overestimates the negative lobe. Besides, the XMCD at the $M_{5}$ edge possesses an additional small positive high energy shoulder which is not reproduced in the theoretical calculations.

\section{References}

[1] X.-L. Qi, S.-C. Zhang,Physics Today 63, 33 (2010).

[2] L. Fu, C. L. Kane, Phys. Rev. Lett. 100, 096407 (2008).

[3] R. Yu, W. Zhang, H.-J. Zhang, S.-C. Zhang, X. Dai, Z. Fang, Science 329, 61 (2010).

[4] L.J. Collins-McIntyre, M.D. Watson, A.A. Baker, S.L. Zhang, A.I. Coldea, S.E. Harrison, A. Pushp, A.J. Kellock, S.S. P. Parkin, G. van der Laan, T. Hesjedal, AIP Adv. 4, 127136 (2014).

[5] L.J. Collins-McIntyre, S.E. Harrison, P. Schoenherr, N.J. Steinke, C.J. Kinane, T.R. Charlton, D. AlbaVeneroa, A. Pushp, A.J. Kellock, S.S.P. Parkin, J.S. Harris, S. Langridge, G. van der Laan, T. Hesjedal, Europhys. Lett. 107, 57009 (2014).

[6] T. Chen, W. Liu, F. Zheng, M. Gao, X. Pan, G. van der Laan, X. Wang, Q. Zhang, F. Song, B. Wang, B. Wang, Y. Xu, G. Wang, R. Zhang, Adv. Mater. 27, 4823 (2015).

[7] V.N. Antonov, M. Galli, F. Marabelli, A.N. Yaresko, A.Y. Perlov, E. Bauer, Phys. Rev. B 62, 1742 (2000).

[8] V.N. Antonov, B.N. Harmon, A.N. Yaresko, Phys. Rev. B 63, 205112 (2001).

[9] V.N. Antonov, L. Uba, S. Uba, A.N. Yaresko, A.Y. Perlov, V.V. Nemoshkalenko, Low Temp. Phys. 27, 425 (2001).

[10] V.N. Antonov, O. Jepsen, A.N. Yaresko, A.P. Shpak, J. Appl. Phys. 100, 043711 (2006).

[11] V.P. Antropov, V.N. Antonov, L.V. Bekenov, A. Kutepov, G. Kotliar, Phys. Rev. B 90, 054404 (2014).

[12] L. Uba, S. Uba, A. Yaresko, A. Perlov, V. Antonov, R. Gontarz, J. Magn. Magn. Mater. 193, 159 (1999).

[13] A.Y. Perlov, A.N. Yaresko, V.N. Antonov, PYLMTO, A Spin-polarized Relativistic Linear Muffin-tin Orbitals Package for Electronic Structure Calculations (unpublished).

[14] J.P. Perdew, K. Burke, M. Ernzerhof, Phys. Rev. Lett. 77, 3865 (1996).

[15] V.V. Nemoshkalenko, A.E. Krasovskii, V.N. Antonov, V.N. Antonov, U. Fleck, H. Wonn, P. Ziesche, Phys. Status Solidi (b) 120, 283 (1983).

[16] A.H. MacDonald, W.E. Pickett, D.D. Koelling, J. Phys. C 13, 2675 (1980).

[17] R.W. Wyckoff, Crystal Structures, Vol. 2, Wiley, New York 1964.

[18] P. Rakyta, B. Ujfalussy, L. Szunyogh, New J. Phys. 17, 123011 (2015).

[19] T. Förster, P. Krüger, M. Rohlfing, Phys. Rev. B 93, 205442 (2016). 